\title{
Aportes hacia la construcción de un perfil docente para el primer año: la importancia del vínculo empático, el valor académico y el trabajo colaborativo
}

\author{
Carlos Alberto Acevedo Cossio \\ Orcid: 0000-0001-6504-8230 \\ carlos.acevedo@inacapmail.cl \\ INACAP, Sede Concepción-Talcahuano
}

\section{Resumen}

El objetivo de este estudio fue determinar las competencias básicas que deben poseer los docentes que realizan clases a estudiantes que ingresan a la enseñanza superior, principalmente aquellas que generan compromiso académico en los alumnos, con la finalidad de facilitar la progresión y participación de estos en sus procesos de estudios para así alcanzar los aprendizajes esperados para cada carrera.

Se llevó a cabo un método mixto con alcance descriptivo, utilizando el diseño de triangulación concurrente para reflejar algunas de las variables detectadas mediante la aplicación de cuestionarios estandarizados y entrevistas semiestructuradas para rescatar las percepciones de los académicos sobre su quehacer. La muestra estuvo constituida por académicos identificados como actores clave para el objetivo de este estudio $(\mathrm{N}=17)$.

Los resultados obtenidos fueron analizados utilizando el software Atlas.ti 6.2 hasta establecer las categorias que engloban la experiencia de los docentes, y mediante el programa SPSS para obtener frecuencias en términos de los puntajes de escalas que midieron las variables Liderazgo, Empatía y Compromiso Académico. Los resultados evidencian que el perfil del Académico de primer año debe contemplar el Vínculo empático, Trabajo colaborativo, Valor académico y la Percepción de competencia. 
Palabras clave: perfil docente, educación superior, docente transversal, perfil docente inicial.

\section{Abstract}

The objective of this study was to determine the basic competencies that teachers who teach students entering higher education should possess, mainly those that generate academic commitment in the students, with the purpose of facilitating their progression and participation in their study processes in order to achieve the learning expected for each career.

A concurrent triangulation design with descriptive scope was used, with a mixed method to reflect some of the variables detected through the application of questionnaires and semi-structured interviews. The sample consisted of firstyear teachers identified as key actors for the purpose of this study ( $\mathrm{N}=17)$.

The results obtained were analyzed using the Atlas.ti 6.2 software to establish the categories that encompass the teachers' experience, and SPSS program to obtain frequencies of the scale scores that measured the variables Leadership, Empathy and Academic Engagement. The results suggest that the profile of the first-year teacher must contemplate the Empathic link, Collaborative work, Academic value and the Perception of competence.

Key words: teacher's profile, higher education, transverse teacher, initial teacher's profile.

\section{Problema y justificación}

En este trabajo se presentan los esfuerzos realizados por delimitar las competencias que deben poseer aquellos profesores que imparten clases a estudiantes noveles, especialmente, en asignaturas de introducción a estudios superiores, cuyo principal objetivo es entregar herramientas de autogestión, autocontrol, gestión de tiempo y compromiso académico.

Dada la variedad de perfiles de académicos que imparten clases en primer año de carrera, se consideró relevante llevar a cabo este estudio, cuyo objetivo era «determinar las competencias básicas que deben poseer los docentes que realizan clases a estudiantes que ingresan a estudios superiores».

Como se ha planteado en diversos estudios sobre perfil docente, su objetivo es promover una formación integral del estudiante, tanto en su formación humana, valórica y ética como en 
la vertiente académica propia de la especialidad que este estudie. Para esto el profesor requerirá de ciertas habilidades genéricas como la escucha activa y el reconocimiento afectivo propio y de sus estudiantes como elemento clave, velando al mismo tiempo por el cumplimiento de gestiones y normativas institucionales (Tobón, 2014; Manota y Melendro, 2015; Parra, Tobón y López, 2015).

Sin embargo, la academia actualmente se da en un contexto en que los docentes, llevados por las transformaciones del mundo laboral, no poseen una militancia definida con una sola institución y sus objetivos, sino que transitan en diversas casas de estudio para conseguir su subsistencia académica y social, lo cual no asegura un compromiso efectivo con su quehacer en un espacio determinado (González-Burboa y Acevedo, 2016).

Por ello, se vuelve necesario analizar la percepción del docente respecto a qué es lo que resulta más relevante para desempeñar su rol y establecer espacios que permitan, desde el involucramiento académico, lograr la sinergia entre estudiante, docente y currículum (Cañedo y Figueroa, 2013; Flynn, 2014).

\section{Marco teórico}

Con el aumento de la demanda por educación superior, explotada en Chile desde 1990 mediante las políticas instauradas que hicieron posible la propagación de universidades privadas, también surge un aumento de la demanda por profesionales que cumplan con formar a los educandos en las distintas áreas de su competencia. La evidencia sugiere que los docentes universitarios, reducidos por la visión de negocios y el nuevo orden jurídico a la categoría de profesionales independientes, se afanan en conseguir su subsistencia académica y social en una sociedad que está cambiando sus reglas, sus modos de conocimiento y sus formas de organización (Esquivel, 2007).

Ante este panorama, se observa la necesidad de atender a las características que tienen los docentes que se transforman en el primer rostro que recibe el estudiante por parte de la institución, de la carrera y, muchas veces, de su experiencia en educación superior (Hernández-Sellés, González-Sanmamedy y Muñoz-Carril, 2015). 
La práctica docente requiere de una sólida preparación y conocimiento de la disciplina que se debe complementar con la constante vigencia del contenido que se imparte (Zabalza y Beraza, 2003). Pero, además, uno de los mayores desafíos que enfrenta un docente supone que enseñar no se reduce meramente a lo curricular o lo didáctico. Por el contrario, se asume que el docente "reflexiona, analiza e interpreta aspectos intelectuales y afectivos, propios y de sus estudiantes, y en función de ello, realiza su práctica docente» (Cañedo y Figueroa, 2013: 2).

En este contexto, las concepciones sobre el quehacer académi$\mathrm{co}$, en particular, sobre los modelos de liderazgo vigentes, como el modelo de liderazgo transformacional (Hermosilla, Amutio, Costa y Páez, 2016) o el modelo de liderazgo delta (Jordan y Garay, 2009) y su aplicación en el ámbito educativo en el modelo de liderazgo pedagógico delta (Cifuentes y Acevedo, 2017), dan cuenta de lo relevante que son las competencias con las que cuenta el docente para desenvolverse, tanto en el interior del aula como en todos los espacios relacionales en que se involucra con el estudiante; y aquel es, por una parte, el organizador y moderador de los procesos de aprendizaje en el contexto de la sala de clases, y, por otra, una imagen que seguir desde el puesto de experticia que le brinda su rol, vindicando la importancia de la acción observada (modelada) como principal insumo que condiciona la acción esperada como respuesta por parte del estudiante.

Existe un amplio espacio de investigación sobre las características que presentan los académicos universitarios que logran impactar significativamente -y de manera positiva- en la vida académica de sus estudiantes (Parra, Tobón y López, 2015); sin embargo, la experiencia indica que la dificultad radica en llevar a los actores educativos a un espacio de apertura a la crítica y flexibilidad frente a los cambios necesarios de introducir en la propia práctica.

Para esto, es imprescindible definir un perfil para el profesorado de primer año, que asegure la creación de un ambiente de aula específico para el aprendizaje, pero además se requiere que las herramientas y recursos implementados durante esta primera etapa formativa en la educación superior se adecuen, desde una mirada socioformativa, a las necesidades de cada carrera en términos de orientación hacia un perfil de egreso y que desafíe a 
los aprendices con estrategias y metas que apunten al desarrollo de competencias (Tobón, 2014). Así, en definitiva, la demanda que se le realiza al académico consiste en un trabajo sistemático para la organización de los contenidos y las estrategias a utilizar desde una perspectiva integrativa, que considere el perfil de ingreso del estudiante y la ubicación de los aprendizajes esperados en la malla curricular para permitir su propensión gradual hacia un mecanismo instruccional que se apoya en la autonomía y el compromiso del individuo con su propio proceso formativo (Acevedo, Cifuentes y Espinoza, 2019).

\section{Método de investigación}

\subsection{Diseño}

Se utilizó un diseño de triangulación concurrente que busca integrar datos cualitativos y cuantitativos para corroborar o elaborar cuerpos de conocimiento mediante el análisis de los datos (Pereira, 2011). Para esto se usó como herramienta la entrevista cuyos datos arrojados fueron tratados de acuerdo con la técnica de análisis de contenido; y la aplicación de 3 cuestionarios para evaluar las variables emergentes desde el análisis bibliográfico que complementan lo declarado: Cuestionario de Compromiso Académico (Peña, Cañoto y Angelucci, 2017), Índice de Reactividad Interpersonal (Fernández, Dufey y Kramp, 2011) y Cuestionario de Estilo y Eficacia del Liderazgo (Montilla, 2013).

\subsection{Participantes}

En cuanto a la selección de la muestra, se utilizó un muestreo discrecional, que permite la selección de individuos de acuerdo con su disponibilidad, la calidad de actores clave y otros criterios previamente establecidos en pos de obtener una mayor riqueza, profundidad y calidad de la información que describa el fenómeno (Strauss y Corbin, 2014).

Los participantes identificados como informantes clave para esta investigación consistieron en profesores que se encontraban realizando clases a estudiantes de primer año de carrera en una asignatura denominada Autogestión, de corte genérico, durante 
el primer semestre del 2018, en una institución de educación superior chilena.

La muestra quedó finalmente conformada por un total de 17 académicos. La edad promedio de la muestra fue de 39 años y en cuanto a género, la muestra se presenta con un $64,7 \%(\mathrm{~N}=11)$ de mujeres y $35,3 \%(\mathrm{~N}=6)$ hombres.

\subsection{Técnicas e instrumentos de recolección de datos}

Las técnicas de recolección de datos utilizadas fueron la entrevista y la aplicación de tres escalas: el Índice de Reactividad Interpersonal, el Cuestionario de Involucramiento Académico Docente y el Cuestionario de Estilo y Eficacia del Liderazgo.

Se construyó y realizó la validación de experto para la entrevista semiestructurada orientada a indagar la atribución de significado sobre la educación, creencias sobre la influencia de la experiencia en la práctica docente, percepción de los docentes sobre los procesos académicos y las prácticas pedagógicas que llevan a cabo en el aula.

Para el Índice de Reactividad Interpersonal (IRI), en cuanto a su consistencia interna, el estadístico alfa de Cronbach ofrece valores adecuados tanto para la dimensión de Angustia Personal $(\alpha=.73)$ como para las de Fantasía $(\alpha=.72)$, Toma de Perspectiva $(\alpha=.68)$ y Preocupación Empática $(\alpha=.65)$.

En cuanto al Cuestionario de Involucramiento Académico Docente, elaborado por Peña (2015), la confiabilidad de la encuesta como un todo, estimada mediante el coeficiente alfa de Cronbach, corresponde a .938, lo cual significa una alta consistencia interna del cuestionario. El Cuestionario de Estilo y Eficacia del Liderazgo (Montilla, 2013) se basa en la teoría del liderazgo situacional de Hershey y Blanchard (1977) y ha sido validada para el trabajo con académicos en Chile (Acevedo, Navarrete y Campos, 2016).

\subsection{Análisis de datos}

El tratamiento de la información recogida mediante las entrevistas consideró la saturación de los datos con 7 casos, ya que las siguientes entrevistas no arrojaron información novedosa sobre el método de comparación constante, y se realizó utilizando el 
software Atlas.ti para la codificación de los datos y la emergencia de las categorías identificadas (Glaser y Strauss, 1967). Se establecieron estas categorías basadas en el discurso de los participantes utilizando temas como unidades de registro que fueron formuladas a través de las tendencias encontradas a partir de los objetivos de los análisis establecidos, y que luego fueron contrastados con el nivel de desarrollo de las variables identificadas mediante software SPSS 24 para obtener frecuencias porcentuales mediante una análisis de estadísticos descriptivos de las respuestas entregadas por los docentes en las escalas aplicadas.

\section{Resultados}

\subsection{Análisis de las entrevistas realizadas}

El análisis de las entrevistas dio como resultado 4 categorías principales, que visibilizan las creencias de los académicos respecto a su quehacer docente en primer año (tabla 1). Estas categorías, están compuestas por un total de 623 citas correspondientes a 161 códigos, agrupados en 27 subcategorías o dimensiones establecidas en función de los objetivos para la extracción de información.

Tabla 1. Codificación de respuestas de entrevistas a los académicos.

\begin{tabular}{|c|c|c|c|c|c|}
\hline Dimensiones/descripción & $f$ & $\%$ & Subcategoría & $f$ & $\%$ \\
\hline A. Caracteristicas del rol & \multirow{6}{*}{204} & \multirow{6}{*}{32,74} & 1. Rol docente & 79 & 38,7 \\
\hline $\begin{array}{l}\text { Descripción de las funcio- } \\
\text { nes y objetivos que con- } \\
\text { templa el rol docente. }\end{array}$ & & & $\begin{array}{l}\text { 2. Objetivos de la práctica pe- } \\
\text { dagógica }\end{array}$ & 53 & 26 \\
\hline \multirow{4}{*}{$\begin{array}{l}\text { Aborda la visión que po- } \\
\text { seen los académicos en } \\
\text { relación con su desempeño } \\
\text { y objetivos, identificando } \\
\text { la posición que ocupa en el } \\
\text { vínculo con el estudiante y } \\
\text { las motivaciones que deter- } \\
\text { minan el quehacer docente. }\end{array}$} & & & $\begin{array}{l}\text { 3. Trabajo colaborativo do- } \\
\text { cente }\end{array}$ & 21 & 10,3 \\
\hline & & & $\begin{array}{l}\text { 4. Motivación hacia la aca- } \\
\text { demia }\end{array}$ & 18 & 8,8 \\
\hline & & & 5. Vínculo docente-estudiante & 16 & 7,8 \\
\hline & & & 6. Necesidades del rol & 14 & 6,9 \\
\hline
\end{tabular}




\begin{tabular}{|c|c|c|c|c|c|}
\hline Dimensiones/descripción & $f$ & $\%$ & Subcategoría & $f$ & $\%$ \\
\hline \multirow[t]{2}{*}{ B. Gestión académica } & \multirow{9}{*}{195} & \multirow{9}{*}{31,3} & 1. Estrategia pedagógica & 58 & 29,7 \\
\hline & & & 2. Formación pedagógica & 50 & 25,6 \\
\hline \multirow{2}{*}{$\begin{array}{l}\text { Descripción de los proce- } \\
\text { sos llevados a cabo para la } \\
\text { planificación e implemen- } \\
\text { tación de las clases. }\end{array}$} & & & 3. Estructura de la clase & 24 & 12,3 \\
\hline & & & $\begin{array}{l}\text { 4. Evidencia del desempeño } \\
\text { docente }\end{array}$ & 16 & 8,2 \\
\hline \multirow{5}{*}{$\begin{array}{l}\text { Aborda la formación peda- } \\
\text { gógica y disciplinar, ade- } \\
\text { más del uso de recursos y } \\
\text { consideraciones en la toma } \\
\text { de decisiones para la eje- } \\
\text { cución de la labor docente. }\end{array}$} & & & 5. Material didáctico & 12 & 6,2 \\
\hline & & & 6. Selección de contenidos & 11 & 5,6 \\
\hline & & & 7. Dificultades & 10 & 5,1 \\
\hline & & & 8. Planificación & 9 & 4,6 \\
\hline & & & $\begin{array}{l}\text { 9. Formación en especiali- } \\
\text { dad }\end{array}$ & 5 & 2,6 \\
\hline \multirow{3}{*}{$\begin{array}{l}\text { C. Contexto académico } \\
\text { Señala elementos relativos } \\
\text { a las condiciones institu- } \\
\text { cionales en que se realiza } \\
\text { el proceso de enseñanza- } \\
\text { aprendizaje. }\end{array}$} & \multirow{5}{*}{142} & \multirow{5}{*}{22,79} & $\begin{array}{l}\text { 1. Diferencias por nivel for- } \\
\text { mativo }\end{array}$ & 54 & 38 \\
\hline & & & $\begin{array}{l}\text { 2. Caracteristicas de los es- } \\
\text { tudiantes }\end{array}$ & 27 & 19 \\
\hline & & & $\begin{array}{l}\text { 3. Criterio de selección do- } \\
\text { cente }\end{array}$ & 24 & 16,9 \\
\hline \multirow{2}{*}{$\begin{array}{l}\text { Involucra la percepción do- } \\
\text { cente en cuanto a las carac- } \\
\text { teristicas que identifican al } \\
\text { estudiante y la relación que } \\
\text { establece el académico con } \\
\text { la institución. }\end{array}$} & & & 4. Sello institucional & 24 & 16,9 \\
\hline & & & 5. Apoyo institucional & 13 & 9,15 \\
\hline D. Experiencias docentes & \multirow{7}{*}{79} & \multirow{7}{*}{12,68} & 1. Antecedentes de docencia & 23 & 29,1 \\
\hline \multirow{2}{*}{$\begin{array}{l}\text { Presenta los referentes que } \\
\text { determinan la práctica do- } \\
\text { cente. }\end{array}$} & & & 2. Experiencias en docencia & 17 & 21,5 \\
\hline & & & 3. Expectativas del docente & 13 & 16,5 \\
\hline \multirow{4}{*}{$\begin{array}{l}\text { Incluye elementos biográfi- } \\
\text { cos y experiencias previas, } \\
\text { además de predisposiciones } \\
\text { y concepciones hacia la la- } \\
\text { bor docente que emergen } \\
\text { desde su propia historia y } \\
\text { de la relación con la insti- } \\
\text { tución. }\end{array}$} & & & 4. Proyección académica & 13 & 16,5 \\
\hline & & & 5. Antecedentes laborales & 8 & 10,1 \\
\hline & & & 6. Definición de docencia & 3 & 3,8 \\
\hline & & & 7. Valoración & 5 & 6,33 \\
\hline
\end{tabular}

\subsection{Análisis de cuestionarios}

\subsubsection{Cuestionario de Estilo y Eficacia del Liderazgo} En relación con los resultados de este cuestionario, se puede plantear que el estilo predominante en los académicos de la asigna- 
tura de Autogestión corresponde al estilo integrado (tabla 2), seguido por el estilo relacionado $y$, finalmente, el estilo autoritario.

Tabla 2. Frecuencia de estilo de liderazgo en docentes de primer año.

\begin{tabular}{lcccccccc}
\hline Estilo de & \multicolumn{8}{c}{$f / \%$} \\
\cline { 2 - 9 } liderazgo & Principal & $\%$ & Secundario & $\%$ & Terciario & $\%$ & Cuarto & $\%$ \\
\hline Relacionado & 5 & $29,41^{*}$ & 12 & 70,59 & 0 & & 0 \\
\hline Integrado & 13 & $76,47^{*}$ & 2 & 11,76 & 2 & 23,53 & 0 \\
\hline Separado & 0 & & 0 & & 1 & 5,88 & 16 & 94,12 \\
\hline Autocrático & 0 & & 4 & 23,53 & 11 & 64,71 & 2 & 23,53 \\
\hline
\end{tabular}

*La sumatoria no es necesariamente complementaria, pues existen casos de coocurrencia en el estilo principal.

En cuanto a la capacidad de adaptabilidad del liderazgo, los resultados del cuestionario basado en el análisis planteado por sus autores se señalan en la tabla 3 .

Tabla 3. Grado de adaptabilidad del liderazgo para los docentes.

\begin{tabular}{lccccc}
\hline Grado de adaptabilidad & Mínimo & Máximo & $\mathrm{f}$ & $\%$ & $\mathrm{M}$ \\
\hline Alto & - & - & 0 & - & - \\
\hline Moderado & 24 & 29 & 13 & 76,5 & 25,92 \\
\hline Bajo & 22 & 23 & 4 & 23,5 & 22,25 \\
\hline
\end{tabular}

Estos resultados indican que no existe ningún académico dentro de la muestra que presente grado alto de flexibilidad en el liderazgo, es decir, la capacidad de adaptar adecuadamente el estilo de liderazgo a las situaciones que se enfrentan; un 76,5\% de la muestra se encuentra en un nivel moderado de adaptabilidad y un $23,5 \%$ de los académicos de la asignatura se encuentran en un nivel disminuido.

\subsubsection{Cuestionario de Involucramiento Académico}

En cuanto a los niveles de involucramiento señalado por los docentes, la información estadística se utiliza en términos de frecuencia para identificar la distribución de los datos. Se puede apreciar una media total de la escala de $(\overline{\mathrm{X}}=182,59 ; \mathrm{SD}=15,08)$, también se presentan los valores mínimos y máximos para este indicador, junto a los valores promedio para cada subescala del 
cuestionario. Estos resultados se reportan en la tabla 4, que se presenta a continuación.

Tabla 4. Distribución de medias del Cuestionario de Involucramiento Académico para una muestra de docentes de la asignatura Autogestión.

\begin{tabular}{llclll}
\hline Indicador & $\mathrm{N}$ & $\mathrm{M}$ & $\mathrm{SD}$ & Mínimo & Máximo \\
\hline Escala & 17 & 182,59 & 15,08 & 158 & 204 \\
\hline Apego a la institución & 17 & 5,20 & 0,65 & & \\
\hline Camaradería & 17 & 5,34 & 0,44 & \\
\hline Contribución & 17 & 4,75 & 0,63 & \\
\hline Impacto & 17 & 5,38 & 0,62 & \\
\hline Imagen & 17 & 5,12 & 0,88 & \\
\hline Motivación & 17 & 5,34 & 0,65 & \\
\hline Pertenencia & 17 & 5,39 & 0,41 & \\
\hline
\end{tabular}

Se utilizó la prueba Kolmogorov-Smirnov para determinar la uniformidad de la distribución de la muestra y se obtuvo una significancia de $(p>0,05)$ que indica la inexistencia de casos que se escapen a la distribución normal de los casos para la escala global.

\subsection{3 Índice de Reactividad Interpersonal}

El último indicador reportado corresponde a los valores obtenidos por los académicos en términos del desarrollo de empatía. En la tabla 5 se presenta la frecuencia de los resultados en la escala en general y en cada subescala, incluyendo valores máximos y mínimos para la medición global.

Tabla 5. Distribución de medias del Índice de Reactividad Interpersonal para una muestra de docentes de la asignatura Autogestión.

\begin{tabular}{lccccc}
\hline \multicolumn{1}{c}{ Indicador } & $\mathrm{N}$ & $\mathrm{M}$ & $\mathrm{SD}$ & Mínimo & Máximo \\
\hline Escala & 17 & 87,59 & 11,20 & 66 & 103 \\
\hline Fantasía & 17 & 2,96 & 0,70 & & \\
\hline Toma de perspectiva & 17 & 2,75 & 0,67 & & \\
\hline Preocupación empática & 17 & 3,79 & 0,52 & & \\
\hline Angustia personal & 17 & 3,32 & 0,60 & & \\
\hline
\end{tabular}


Los resultados observados señalan una media de $(\overline{\mathrm{X}}=182,59$; $\mathrm{SD}=15,08)$. Además, se reportan los valores máximos y mínimos de la muestra, para la cual, la prueba de Kolmogorov-Smirnov señala una distribución uniforme de los casos.

\section{Conclusiones y discusión}

Para comenzar, se discutirán los elementos críticos a los que se refieren los docentes para definir tanto su rol como la naturaleza de su quehacer, que se aprecian en directa relación con lo planteado en la literatura respecto a los roles docentes (Zabalza y Beraza, 2003; Solar y Días, 2009; Mas y Olmos, 2011; Mas, 2012; Peña, Cañoto y Angelucci, 2017), al señalar como puntos esenciales para un adecuado desempeño académico en el primer año, el hecho de mantener vínculos empáticos que visibilicen las necesidades de los estudiantes, pero además, generando espacios de contención y formación valórica que permita compensar algunas de las competencias de entrada deficientes. Es posible observar la necesidad de reflexión y revisión de estos elementos, a la luz de indicadores que señalan que el promedio más bajo en las subescalas corresponde precisamente a la habilidad más necesaria en el académico.

Por otro lado, los académicos coinciden en que la enseñanza debe darse en un contexto de alta exigencia, pero también de cercanía y confianza, lo que está en directa relación con los modelos educativos actuales (Flores, 2011), se comprende, entonces, que el $76,4 \%$ de los académicos presenten como estilo principal un estilo de liderazgo integrado, aunque es importante observar que el estilo que secunda es el relacionado, dejando fuera en primer año la orientación hacia la tarea lo que, desde la perspectiva de los propios académicos, llevaría a una pérdida en el encuadre que les permita desarrollar inicialmente la autogestión. Desde este parámetro es posible indicar que la utilización de un estilo de liderazgo relacionado podría ser más adecuado en los niveles superiores, el mismo caso del estilo autocrático, señalado en el $23 \%$ de los casos como estilo secundario, pero reportado como una fuente de amenaza para los estudiantes porque ahuyentaría al alumno que no posee las competencias iniciales desarrolladas en un nivel adecuado. 
En definitiva, se podría señalar que los aspectos más relevantes para el establecimiento de un perfil académico para el primer año corresponden a:

- Vínculo empático: estaría relacionada con la capacidad del académico de generar un interés en el estudiante para lograr en él el desarrollo de competencias de manera autónoma y significativa, desde un rol de facilitador de procesos, y depende de las herramientas y recursos pedagógicos y relacionales con los que cuente el académico, lo que en primer año sería un nodo crítico.

- Trabajo colaborativo: la capacidad que presente el académico de lograr un trabajo en redes colaborativas con los demás estamentos de la institución, tanto colegas académicos como administrativos. Se relaciona directamente con el estilo y eficacia del liderazgo pedagógico, pues también aborda las pautas relacionales que establece el académico y que transmite a sus estudiantes.

- Valor académico: si el objetivo es el desarrollo de competencias que tengan sentido para la vida del estudiante mediante un proceso de mediación de experiencias vinculantes, debe existir una valoración de los procesos llevados a cabo, comenzando por el académico, para poder transmitirlo a los estudiantes. Este aspecto se relaciona directamente con el compromiso académico y la percepción que tiene tanto de su rol como de la institución de la que forma parte y de sus características.

- Percepción de competencia: referido a las habilidades que el académico percibe que posee para enfrentar los desafíos formativos que se presentan en los cursos nivelatorios de primer año. Se comprende que el estudiante requiere desarrollar habilidades desde distintos niveles iniciales, lo que implica la necesidad de contar con una amplia variedad de herramientas para facilitar el ajuste a estos diversos requerimientos.

Los hallazgos presentados ni por mucho pretenden agotar la temática estudiada, sino más bien plantear como referente la necesidad de analizar las demandas, el contexto y las percepciones que tienen los académicos de primer año, en esta instancia de vinculación inicial entre la institución y el estudiante. En este sentido, cabe la posibilidad de continuar esta línea de investi- 
gación, indagando sobre la percepción de autoeficacia que poseen los académicos de primer año y cómo esto impacta en sus estudiantes.

Se espera que este trabajo sea parte de un impulso que se enfoque en generar una cultura orientada hacia el involucramiento académico desde la perspectiva en que, si una comunidad de aprendizaje es capaz de armonizar sus pautas relacionales, obtendrá claridad respecto de sus objetivos comunes esenciales $y$, solo entonces, podrá enfrentarse al proceso formativo como un referente de calidad en una arquitectura de aprendizaje sistémica.

\section{Referencias bibliográficas}

Acevedo, C.; Cifuentes, C.; Espinoza, M. (2019). Análisis institucional a nivel sede sobre el Involucramiento Académico Estudiantil y Docente. Santiago: INACAP.

Acevedo, C.; Navarrete, C.; Campos, G. (2016). Estilos de liderazgo que ejercen los docentes en las asignaturas de la línea de formación para la empleabilidad, de la Universidad Tecnológica de Chile, INACAP, sede Concepción-Talcahuano (tesis de pregrado). Universidad Tecnológica de Chile, INACAP, Chile.

Cañedo, T.; Figueroa, A. (2013). «La práctica docente en educación superior: una mirada hacia su complejidad». Sinéctica, 41: 2-18. Recuperado el 4 de octubre de 2018 de: <http://www.scielo.org.mx/ scielo.php?script=sci_arttext\&pid=S1665-109X2013000200004\&lng=es\&tlng=es>.

Corbin, J.; Strauss, A.; Strauss, A. L. (2014). Basics of qualitative research. Sage. Esquivel, J. (2007). "Chile: campo experimental para la reforma universitaria». Perfiles educativos, 29(116): 41-59.

Flynn, D. (2014). «Baccalaureate attainment of college students at 4-year institutions as a function of student engagement behaviors: Social and academic student engagement behaviors matter». Research in Higher Education, 55(5): 467.

Flores, C. (2011) «Involucramiento del profesor universitario: Asignatura pendiente para las instituciones de educación superior». Universidades, 61(50): 79-88.

Hermosilla, D.; Amutio, A.; Costa, S. D.; Páez, D. (2016). «El liderazgo transformacional en las organizaciones: variables mediadoras y 
consecuencias a largo plazo». Revista de Psicología del Trabajo y de las Organizaciones, 32(3): 135-143.

Hernández-Sellés, N.; González-Sanmamedy, M.; Muñoz-Carril, P. C. (2015). «El rol docente en las ecologías de aprendizaje: análisis de una experiencia de aprendizaje colaborativo en entornos virtuales». Profesorado. Revista de Currículum y Formación de Profesorado, 19(2): 147-163.

Hershey, P.; Blanchard, K. H. (1977). «Management of organizational behavior». Prentiss Hall, pp. 236-244.

Jordan, R.; Garay, M. (2009). Liderazgo real de los fundamentos a la práctica. Pearson Educación.

Mas, O. (2012). "Las competencias del profesor universitario: la percepción del alumno, de los expertos y de los propios protagonistas». Revista de docencia universitaria, 10(2): 299-318.

Mas, O.; Olmos, P. (2011). "Competencias docentes para la inclusión del alumnado universitario en el marco del Espacio Europeo de Educación Superior». Revista de Educación Inclusiva, 5(1): 159-174.

Montilla, V. (2013). «Liderazgo e inteligencia emocional». Material de trabajo para Diplomado en Gestión y Liderazgo, Universidad FinisTerrae, Chile. Recuperado de Hershey y Blanchard de la Universidad de Washington.

Parra, H.; Tobón, S.; López, J. (2015). «Docencia socioformativa y desempeño académico en la educación superior». Paradígma, 36(1): 42-55. Recuperado de: <http://ve.scielo.org/scielo.php?script=sci_ arttext\&pid=S1011-22512015000100004\&lng=es\&tlng=es $>$.

Peña, G.; Cañoto, Y.; Angelucci, L. (2017). «Involucramiento académico: una escala». Páginas de Educación, 10(1): 114-136. DOI: <https:// doi.org/10.22235/pe.v10i1.1361>.

Pérez, Z. P. (2011). «Los diseños de método mixto en la investigación en educación: Una experiencia concreta». Revista Electrónica Educare, 15(1): 15-29.

Sánchez, F. L. (2009). Las emociones en la educación. Morata.

Solar, M.; Díaz, C. (2009). «Los procesos de enseñanza-aprendizaje en el aula universitaria: Una mirada desde las creencias de académicos de trabajo social y periodismo». Estudios Pedagógicos, 1: 181-197.

Tobón, S. (2014). Proyectos formativos: teoría y práctica. México: Pearson

Zabalza, M. Á.; Beraza, M. Á. Z. (2003). Competencias docentes del profesorado universitario: calidad y desarrollo profesional. Barcelona: Narcea. 
1. La innovación educativa en el contexto de la

Educación Superior Técnico-Profesional.

Claudio Maregatti Solano, María luisa Arancibia Muñoz, Rosa Eliana Romero Alonso

1. Introducción

2. La transformación de las instituciones de

Educación Superior Técnico-Profesional

3. De las prácticas docentes al saber académico

4. El aporte al conocimiento científico sobre la

Educación Superior Técnico-Profesional . . . . . . . . . 15

5. Referencias bibliográficas .................. . 21

2. Caracterización de estudiantes de Educación Técnico-Profesional respecto a su Educación Secundaria: reflexiones para la Educación Superior

Javier Torres-Vallejos, Juan Ignacio Venegas Muggli, Simón Mundaca Toledo, Juan Carlos Oyanedel Sepúlveda

1. Introducción . . . . . . . . . . . . . . . . . . . 26

2. Metodología ......................... . 30

2.1 Muestra . . . . . . . . . . . . . . . . . . . . . 30

2.2 Variables . . . . . . . . . . . . . . . . . . . . . 32

2.3 Tratamiento de bases de datos . . . . . . . . . . . . 32

2.4 Análisis de datos..................... 33

3. Resultados ........................... 33

3.1 Características educativas y académicas en

Educación Superior ..................... . 34

3.2 Características educativas y académicas del establecimiento secundario de egreso de Enseñanza Media . . . . . . . . . . . . . . . . . . . . 36

4. Discusión y conclusiones. . . . . . . . . . . . . . . 37

5. Referencias bibliográficas ................. . . 39 
3. Aportes hacia la construcción de un perfil docente para el primer año: la importancia del vínculo empático, el valor académico y el trabajo colaborativo

Carlos Alberto Acevedo Cossio

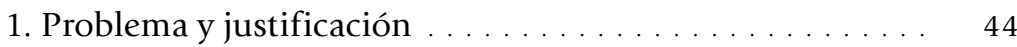

2. Marco teórico ....................... 45

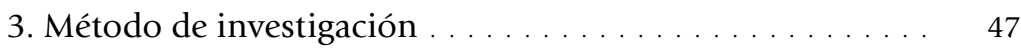

3.1 Diseño. . . . . . . . . . . . . . . . . . . 47

3.2 Participantes...................... 47

3.3 Técnicas e instrumentos de recolección de datos. . . . 48

3.4 Análisis de datos. . . . . . . . . . . . . . . . . . . . . 48

4. Resultados ... . . . . . . . . . . . . . . . . . . . . . . 49

4.1 Análisis de las entrevistas realizadas . . . . . . . . . 49

4.2 Análisis de cuestionarios . . . . . . . . . . . . . 50

4.2.1 Cuestionario de Estilo y Eficacia del Liderazgo . . 50

4.2.2 Cuestionario de Involucramiento Académico . . . 51

4.2.3 Índice de Reactividad Interpersonal . . . . . . . . . 52

5. Conclusiones y discusión ................. . 53

6. Referencias bibliográficas .................. 55

4. Mentoría docente para instalar estrategias de resolución de problemas en matemáticas iniciales

Cristian Iván Ramos Arrepol, Carlos Alberto Acevedo

Cossio

1. Problema y justificación . . . . . . . . . . . . . . . . 58

2. Marco teórico . . . . . . . . . . . . . . . . . . . . . . . 59

2.1 Trabajo colaborativo . . . . . . . . . . . . . . . . . 59

2.2 Mentorías ......................... 60

3. Método de investigación .................... 61

3.1 El perfil de entrada del docente . . . . . . . . . . . 62

3.2 Reclutamiento docente. . . . . . . . . . . . . 62

3.3 La caminata de aula . . . . . . . . . . . . . . . . . . 63

3.3.1 El primer paso . . . . . . . . . . . . . . . . 63

3.3 .2 Segunda ronda . . . . . . . . . . . . . . . . . . 64

3.3.3 Ronda final. . . . . . . . . . . . . . . . . . 64

3.4 Abordaje de situaciones críticas . . . . . . . . . . . . 64

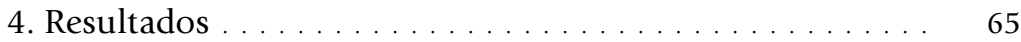

5. Conclusiones y discusión. . . . . . . . . . . . . . . . 69

6. Referencias bibliográficas ................... 70 
5. Clase invertida para la formación inicial de educadoras diferenciales sobre aprendizaje matemático

Laura Marjorie Espinoza Pastén

1. Problema y justificación . . . . . . . . . . . . . . . . . . 74

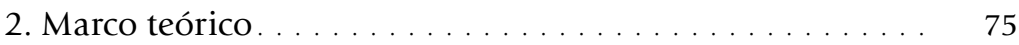

3. Proceso de la innovación. . . . . . . . . . . . . . . 76

3.1 Contexto ........................ 76

3.2 Diseño didáctico . . . . . . . . . . . . . . . 77

4. Resultados ......................... . 80

4.1 Resultados considerando el rendimiento académico. . 80

4.2 Resultados considerando la valoración de las estudiantes $\quad 81$

5. Discusión y conclusiones . . . . . . . . . . . . . . . 83

6. Referencias bibliográficas ................. 84

6. ¿Cómo medir el aprendizaje en innovación? Análisis factorial confirmatorio del Innovator's Behavior Questionnaire (i)BQ en universitarios chilenos. . . . . . 87 Jorge Maluenda Albornoz, Pedro Lledó Aninat

1. Problema y justificación . . . . . . . . . . . . . . . . . . . . . 89

2. Marco teórico . . . . . . . . . . . . . . . . . . . . . 90

3. Método............................. 92

4. Resultados ......................... 93

5. Conclusiones y discusión. . . . . . . . . . . . . . . . . 96

6. Referencias bibliográficas ................. 98

7. Programa de inclusión para actores de Educación Superior Técnico-Profesional . . . . . . . . . . . . . . . . 101

Marcela Pérez Poquet, Paulina Muñoz Villalobos, Victoria Aravena Rivas

1. Problema y justificación del estudio . . . . . . . . . . . . 102

2. Marco teórico . . . . . . . . . . . . . . . . . . . . . . 105

3. Metodología de investigación ................ 108

3.1 Descripción del contexto . . . . . . . . . . . . . . . 108

3.2 Participantes. . . . . . . . . . . . . . . . . . . . 109

3.3 Instrumentos y procedimientos . . . . . . . . . . . . . . 109

4. Resultados . . . . . . . . . . . . . . . . . . . . . . . 111

5. Conclusiones y discusión. . . . . . . . . . . . . . . . . . 113

6. Referencias bibliográficas ................. 113

Los autores. . . . . . . . . . . . . . . . . . . . . . . . . ${ }^{115}$ 